\section{Quand un solide cristallin} est martelé ou plié, il peut se déformer de manière irréversible. Cette plasticité des cristaux est essentielle, car elle permet, d'une part, la transformation de lingots métalliques en éléments de structure de formes très diverses et, d'autre part, une déformation douce des matériaux de structure soumis à une surcharge, évitant ainsi leur rupture brutale. Bien que l'origine microscopique de la plasticité cristalline, i.e. les dislocations, ait été découverte

il y a plusieurs décennies, sa modélisation reste un défi important de la physique.

Des avancées significatives ont cependant été réalisées ces dernières années, en particulier grâce au développement de simulations dites mésoscopiques.

\title{
Étudier les propriétés dynamiques des dislocations pour prédire la déformation plastique des cristaux
}

Benoît Devincre (benoit.devincre@onera.fr)

Laboratoire d'Étude des Microstructures, UMR104 CNRS-0NERA, 29 avenue de la division Leclerc, 92322 Chatillon Cedex

Ces vingt dernières années, la recherche en science des matériaux a bénéficié du développement rapide de nouvelles formes de modélisations numériques : les simulations mésoscopiques. La finalité des études utilisant ces modèles est presque toujours la même : il s'agit de construire le lien entre un phénomène élémentaire à l'échelle microscopique, ici la mobilité d'une dislocation, et l'influence globale d'une assemblée de dislocations sur les propriétés du matériau massif. Ce lien, comblant le fossé énorme existant entre modèles microscopiques et macroscopiques, est assuré pour l'essentiel par les techniques de simulations mésoscopiques (aux échelles se situant entre le nanomètre et le micron).

Les modèles microscopiques de la plasticité cristalline sont souvent limités aux seuls aspects atomiques. Ils s'intéressent généralement aux propriétés élémentaires des dislocations et, à quelques exceptions près, n'ont pas de caractère prédictif sur le comportement global du matériau. À l'opposé, les modèles macroscopiques s'articulent autour de la mécanique des milieux continus qui, certes, fournit aux ingénieurs une description quantitative de la plasticité, mais reste bien souvent phénoménologique et n'inclut ni les fluctuations spatiales induites par les microstructures de dislocations ni les longueurs d'échelle associées à ces dernières. Concilier ces deux approches ne peut venir que d'une description de la plasticité à une échelle intermédiaire, dite mésoscopique. À cette échelle, on considère une densité de dislocations suffisamment importante pour étudier leurs propriétés collectives. C'est pourquoi les simulations de dynamique des dislocations (DD) sont maintenant un passage obligé des études «multi-échelles » de la plasticité [1].

Les principes de base des simulations de DD sont les suivants. L'objet élémentaire est ici la dislocation individuelle, représentée d'une manière simplifiée dans un continuum élastique. Les lignes de dislocations sont discrétisées en segments élémentaires, souvent disposés sur un réseau de simulation de même symétrie que le matériau cristallin. Le déplacement des dislocations se fait par sauts discrets dans le continuum élastique et dans des directions imposées par les symétries du cristal. Les propriétés élastiques des dislocations (forces d'interactions, tension de ligne, sollicitations mécaniques) sont décrites dans le cadre de la théorie élastique [2]. Leurs propriétés de " cœur" (c.f. encadré 1), qui sont activées thermiquement, sont modélisées avec des règles locales de simulation décrivant des processus aléatoires thermomécaniques intervenant à l'échelle atomique. Finalement, l'aire balayée par les dislocations après chaque incrément de simulation nous renseigne sur la déformation plastique produite dans le volume simulé.

Parmi les différents aspects de la plasticité cristalline encore mal compris, la question de l'écrouissage plastique (c'està-dire l'augmentation progressive de la force qu'il faut exercer sur un cristal pour en poursuivre la déformation plastique), en relation avec la formation de microstructures de dislocations, est un point délicat. En effet, si des modèles 


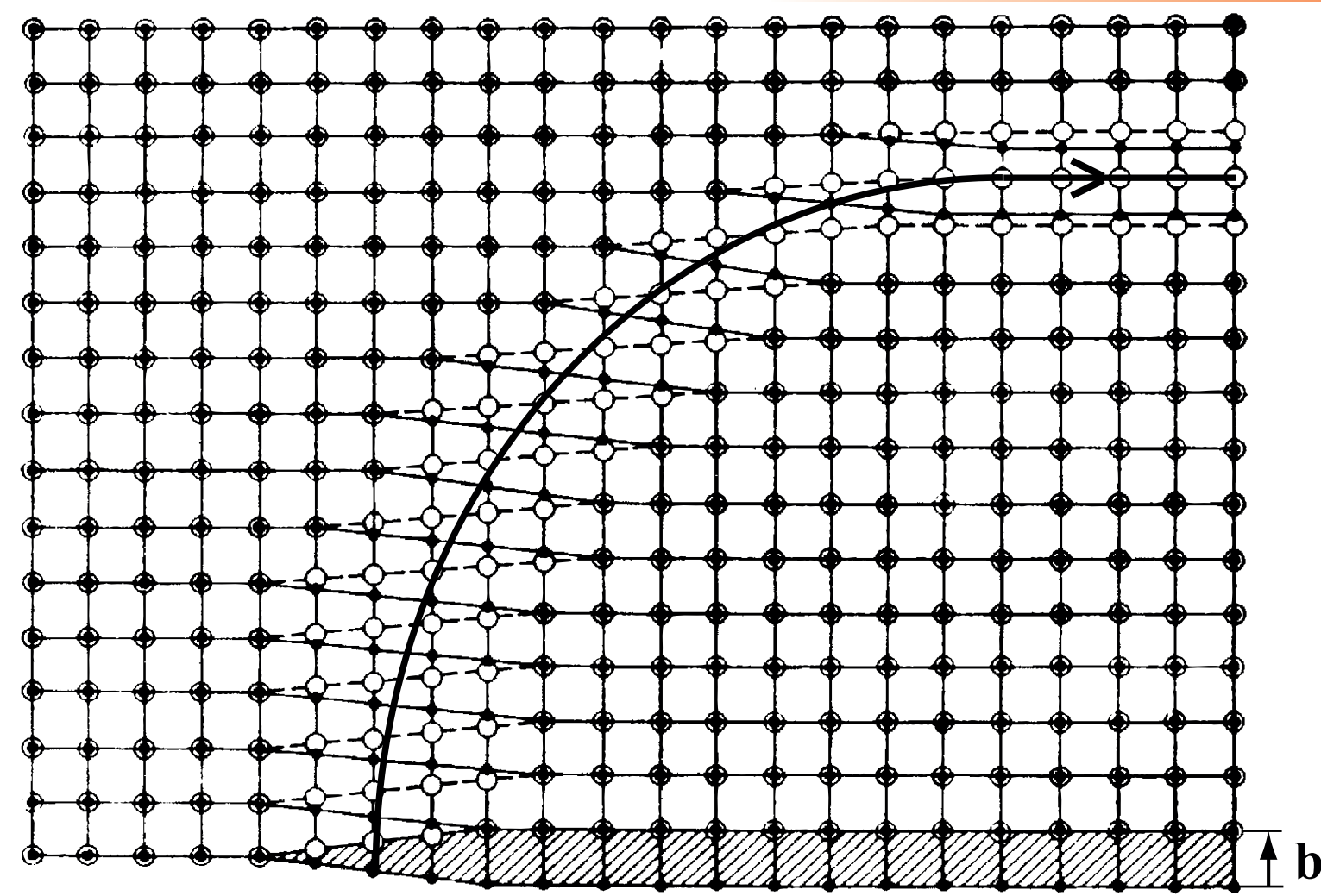

Représentation schématique d'une dislocation formée dans un réseau cubique, avec un cisaillement d'amplitude et de direction définies par le vecteur b (le vecteur de Burgers). La ligne de dislocation représentée par la courbe noire délimite la frontière entre les parties du cristal cisaillée et non cisaillée. Le cisaillement appliqué se manifeste par la formation d'une marche atomique (partie hachurée) en bas et à droite du schéma. Les cercles vides sont les atomes juste au-dessus du plan de coupe et les plus petits points sont les atomes juste en dessous. (D'après W.T. Read, Jr., Dislocations in crystals, McGraw-Hill, 1953.)

\section{Notions sur les dislocations dans un réseau cristallin}

La capacité des solides cristallins à se déformer plastiquement (c'est-à-dire de manière irréversible) est un phénomène lié au mouvement de défauts linéaires du réseau cristallin, appelés les dislocations. On peut se représenter les dislocations, dans leur forme la plus simple, comme la limite d'un demi plan atomique inséré dans un cristal parfait par cisaillement (figure ci-dessus). Ces défauts perturbent I'espacement interatomique et induisent des distorsions à l'origine d'un champ élastique à longue portée, centré autour du cœur des dislocations (fig. E1).

Sous I'action d'une force mécanique appliquée, les dislocations peuvent se déplacer dans certaines directions cristallographiques et ainsi transporter un cisaillement plastique élémentaire favorisant un changement de forme "plastique » du cristal. Au cours de cette déformation, la longueur des lignes des dislocations augmente. Ainsi, après une déformation significative de quelques pourcents, la longueur totale des dislocations dans un mètre cube de métal est typiquement 10 millions de fois la distance de la Terre au Soleil.

De plus, comme les dislocations interagissent entre elles pour former des jonctions (fig. E1) ou interagissent avec divers obstacles cristallins qui les freinent ou les arrêtent, la contrainte qu'il faut imposer pour continuer la déformation d'un cristal augmente continûment. Ainsi, les cristaux se durcissent (s'écrouissent) pendant leur déformation jusqu'à une contrainte maximum avant rupture. Cette dynamique des dislocations est un phénomène physique complexe, contrôlant les propriétés mécaniques des matériaux.

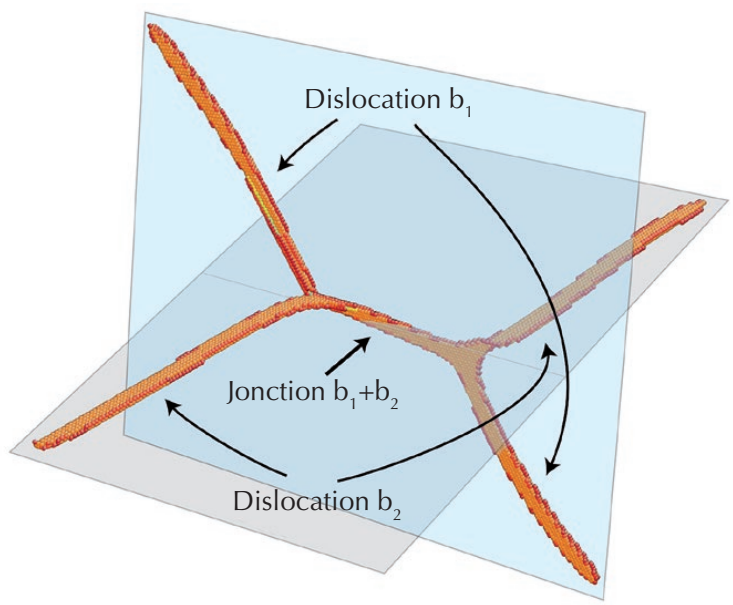

E1. On peut visualiser avec une simulation atomique les dislocations contenues dans un cristal, en coloriant les atomes déplacés de quelques pourcents vis-à-vis de leur position de référence dans le cristal parfait. On fait ainsi apparaître le « cœur » des dislocations (en orange sur la figure). Certaines propriétés importantes des dislocations, telles que la mobilité ou la possibilité de changer de plan cristallographique de déplacement, sont contrôlées par des réarrangements atomiques locaux intervenant dans cette région du cœur. L'énergie élastique associée aux dislocations en dehors du cœur étant très grande, il peut être favorable localement de combiner le champ élastique de deux dislocations pour former une nouvelle ligne de dislocation de vecteur de Burgers $\mathbf{b}_{1}+\mathbf{b}_{2}$. La mobilité de cette nouvelle dislocation étant très réduite, on parle alors de dislocation jonction. L'accumulation de ces jonctions pendant la déformation est un mécanisme essentiel de l'écrouissage plastique, appelé durcissement de la forêt. (D’après D. Rodney.) 

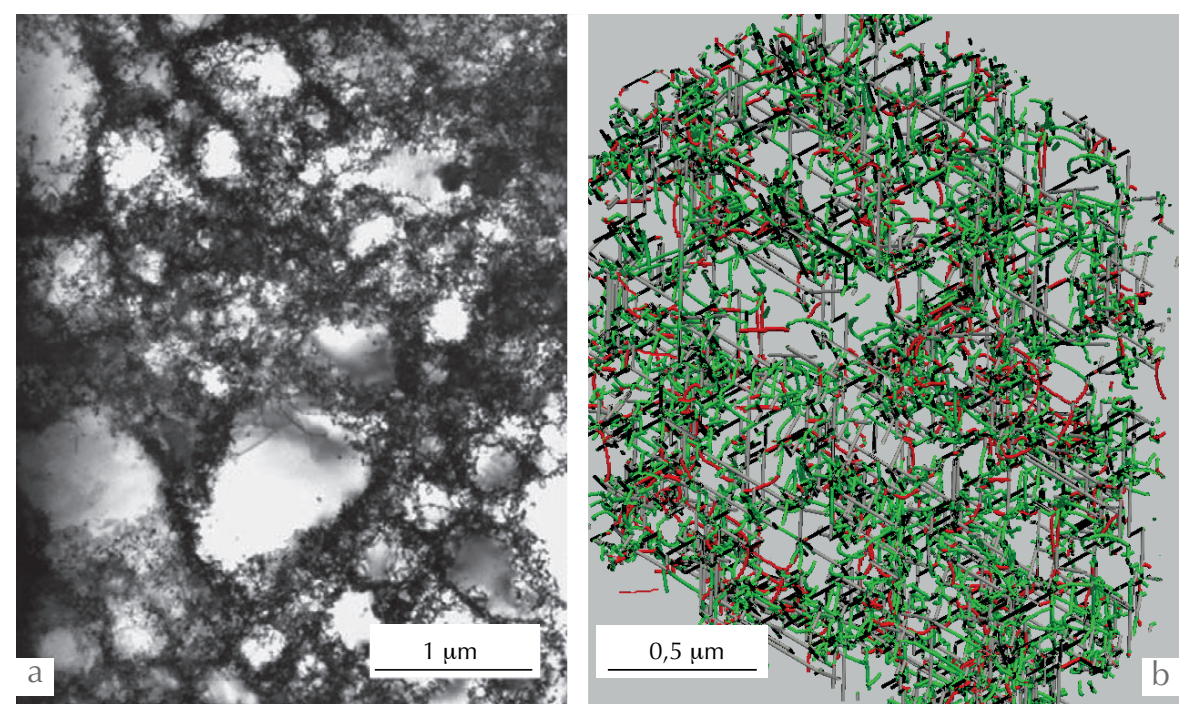

1. Microstructures de dislocations.

(a) Image par microscopie électronique en transmission d'une microstructure de dislocations dite " cellulaire », observée dans un monocristal de cuivre après 28\% de compression suivant la direction cristalline [001].

Les parois de cellules (en noir) sont constituées d'une grande densité de lignes de dislocations enchevêtrées. Ces parois entourent des volumes cristallins approximativement sphériques, contenant peu ou pas de dislocations (zones claires). (M. Kassner et al., Int. J. Mech. Sci. 100 (2009) 333-339.)

(b) Microstructure de dislocations dans du cuivre, simulée par dynamique des dislocations pour des conditions de déformation et de densité de dislocations similaires à celles de l'échantillon présenté en (a). Les dislocations se déplaçant dans le plan de la tranche illustrée sont tracées en rouge et celles traversant ce plan en vert.

\section{Auto-organisation, invariance d'échelle et loi de puissance}

De nombreux phénomènes physiques comme les glissements de terrain ou les feux de forêt, se manifestent par une complexité structurelle et dynamique importante à partir de lois physiques simples s'exerçant à l'échelle élémentaire. S'il n'existe pas encore de définition générale de la complexité en physique, celle-ci se caractérise souvent sous la forme d'une invariance d'échelle. En effet, les structures complexes étudiées à différents niveaux de grossissement semblent souvent autosimilaires, c'est-à-dire indépendantes de l'échelle d'observation. Mathématiquement, cette invariance d'échelle est révélée à I'aide de lois de puissance de la forme $P(X)=X^{-\beta}$, avec $P(X)$ la densité de probabilité $\mathrm{d}^{\prime}$ une quantité observable $X$, et $\beta$ un exposant d'échelle relié à la dimension fractale du phénomène étudié.

L'existence d'une invariance d'échelle dans un domaine spatial et/ou temporel large implique que la complexité mesurée résulte en réalité d'un seul mécanisme critique contrôlant la structure du phénomène à toutes les échelles. De plus, une propriété commune de ces systèmes est qu'ils peuvent s'organiser spontanément par étapes intermittentes. Lors d'une sollicitation faible, l'énergie donnée au système s'accumule jusqu'au déclenchement d'un phénomène discret permettant la dissipation rapide d'une grande partie de l'énergie accumulée. On désigne généralement ces phénomènes de relaxation comme étant des « avalanches ».

\section{\>}

anciens [2] permettent par exemple de calculer le seuil de déformation plastique des métaux pour un état cristallin donné (essentiellement fixé par la densité des dislocations), les mécanismes responsables de la formation d'une microstructure de dislocations organisée (fig. 1a) et la diminution de sa taille caractéristique en cours de déformation restent un sujet de controverses. La modélisation de ces
Propriétés collectives et avalanches de dislocations

Des études récentes, faisant appel soit à l'expérience soit à la simulation, donnent un nouvel éclairage sur les propriétés collectives des dislocations à l'échelle de la microstructure. La dynamique des dislocations observée à l'échelle mésoscopique n'est pas régulière, mais s'apparente plutôt à des phénomènes d'avalanche. Ainsi, contrairement à une idée communément admise, la déformation plastique n'est en général pas assimilable à un écoulement continu, mais est un phénomène intermittent [3]. Le cristal se déforme par à-coups, d'amplitudes variables et imprévisibles. L'analyse statistique de cette intermittence nous apprend que la déformation plastique appartient ainsi à une classe très générale de systèmes physiques non linéaires, caractérisée par une auto-organisation issue d'un phénomène d'instabilité intermittent (fig. 2). Une signature forte de cette classe de systèmes est une distribution en loi de puissance du phénomène intermittent observé, et qui présente donc une invariance d'échelle (c.f. encadré 2).

On sait maintenant que les avalanches de dislocations sont distribuées en loi de puissance, avec un exposant critique constant d'environ 1,5. Le caractère universel de cet exposant a été vérifié aussi bien dans des matériaux massifs (figs $2 a-b$ ) que dans des échantillons de dimensions submicroniques. Dans tous les cas, chaque incrément de déformation plastique représentant un événement élémentaire des distributions de la figure $2 \mathrm{~b}$ est amorcé par la destruction d'une des jonctions interconnectant, en réseau tridimensionnel, les dislocations dans le cristal. La destruction de cette jonction permet la libération d'une petite section de dislocation mobile qui, via les interconnexions entre dislocations ou (dans une plus faible mesure) des interactions élastiques sans contact, va à son tour libérer un nombre imprévisible d'autres segments de dislocations. Chaque avalanche induit alors une augmentation de la densité de dislocations contenues dans le cristal, par l'extension de la longueur des lignes mobiles en cours de déplacement (fig. 3). Finalement, un incrément de déformation se termine par un découpage des dislocations mobiles en plus petits segments accrochés au réseau 3D de dislocations immobiles obstruant leurs déplacements. La force 
d'ancrage d'un réseau de dislocations augmentant avec la densité de lignes localement enchevêtrées, l'intermittence favorise une accumulation hétérogène des dislocations et donc la formation de microstructures organisées.

Il faut alors noter que la constance de l'exposant critique des distributions d'avalanches de dislocations observée pendant la déformation plastique d'un matériau peut être interprétée comme une signature de sa bonne "santé ", i.e. une indication de la conservation de son intégrité. Sortir du domaine d'invariance d'échelle des avalanches, par exemple en fin de déformation plastique, nous informe sur l'apparition d'un phénomène tierce participant à la déformation qui, généralement, va induire une altération catastrophique du matériau et sa rupture.

\section{Changement d'échelles et durcissement plastique}

Le durcissement plastique des matériaux est une propriété définie à l'échelle globale des essais mécaniques; il suppose une variation continue de la déformation et de la densité de dislocations dans le cristal. C'est donc, par définition, un concept physique mal adapté à l'échelle des avalanches de dislocations. Une reformulation de ce phénomène nécessite alors l'intégration d'un grand nombre d'événements discrets observés dans les microstructures de dislocations, pour concevoir une théorie continue de la plasticité cristalline : ici, les simulations mésoscopiques sont un outil précieux qui permet d'obtenir numériquement, à l'échelle d'un volume élémentaire représentatif, ces valeurs moyennes qui caractérisent les propriétés collectives des dislocations. Par exemple, les simulations permettent de calculer le libre parcours moyen des dislocations, concept statistique qui définit le taux d'accumulation des dislocations pendant la déformation d'un matériau [4]. En accord avec les processus d'avalanches décrits plus haut, il devient clair que le libre parcours moyen des dislocations dépend de trois paramètres ; i) la force motrice exercée sur les dislocations pour les déplacer, ii) la longueur moyenne $l_{0}$ des segments de dislocation immobilisés sur chaque jonction de la microstructure, et iii) $\mathrm{P}_{0}$, le taux de succès de formation d'une jonction à chaque fois que deux lignes de dislocations se croisent. Le calcul de ces quantités avec

\〉>

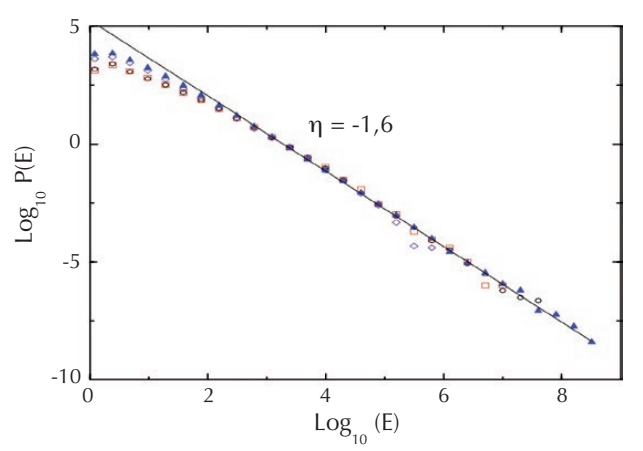

2. Intermittence et loi de puissance.

La déformation plastique des matériaux cristallins est souvent intermittente et contrôlée par des avalanches de dislocations distribuées en loi de puissance. Ces distributions d'avalanches manifestent une invariance d'échelle, avec un exposant $\eta$ remarquablement constant sur un domaine borné généralement par la sensibilité des moyens expérimentaux d'un côté et les dimensions du cristal aux grandes tailles

a d'avalanches.

(a) Distributions de l'énergie d'émission acoustique (proportionnelle à la déformation plastique) mesurées dans des monocristaux massifs de glace en cours de déformation (M.-C. Miguel et al., Nature, 410 (2001) 667). (b) Distributions d'incrément de déformation plastique simulées par DD pour des monocristaux de cuivre déformés suivant différentes orientations cristallines (B. Devincre et al., Science 320 (2008) 1745).
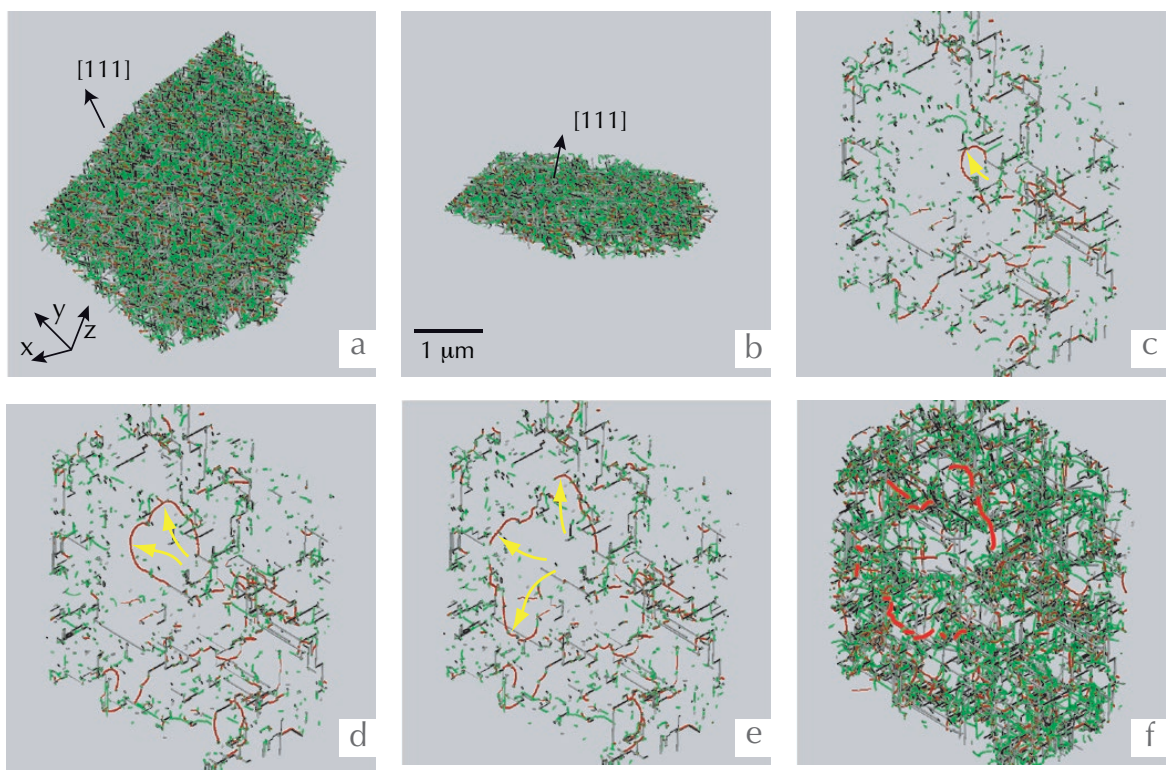

\section{Avalanches de dislocations.}

Simulation par dynamique des dislocations de la déformation plastique d'un volume de cuivre monocristallin contenant une grande densité de dislocations (a). On extrait de ce volume une tranche d'épaisseur 0,05 um au centre, et de direction normale \{111\} (b). Les dislocations se déplaçant dans la tranche sont tracées en rouge et celles la traversant sont en vert. Sur ces images réalisées avec des intervalles de temps espacés de 0,1 us (c-e), on voit que la distribution de dislocations dans le plan central de la tranche est essentiellement immobile en cours de déformation. De temps à autre, on observe l'extension rapide d'un segment (flèche jaune sur la figure c). (e segment de dislocation se déplace ensuite en interagissant avec le réseau 3D de dislocations obstruant sa trajectoire (d-e). Après une aire balayée plus ou moins grande, l'avalanche de dislocations s'arrête dans des enchevêtrements de dislocations formant des parois de plus en plus difficiles à franchir. La microstructure de dislocation se formant progressivement (ici, après plusieurs milliers d'avalanches) est montrée en (f), en visualisant les 0,05 $\mu$ m d'épaisseur de la tranche. 


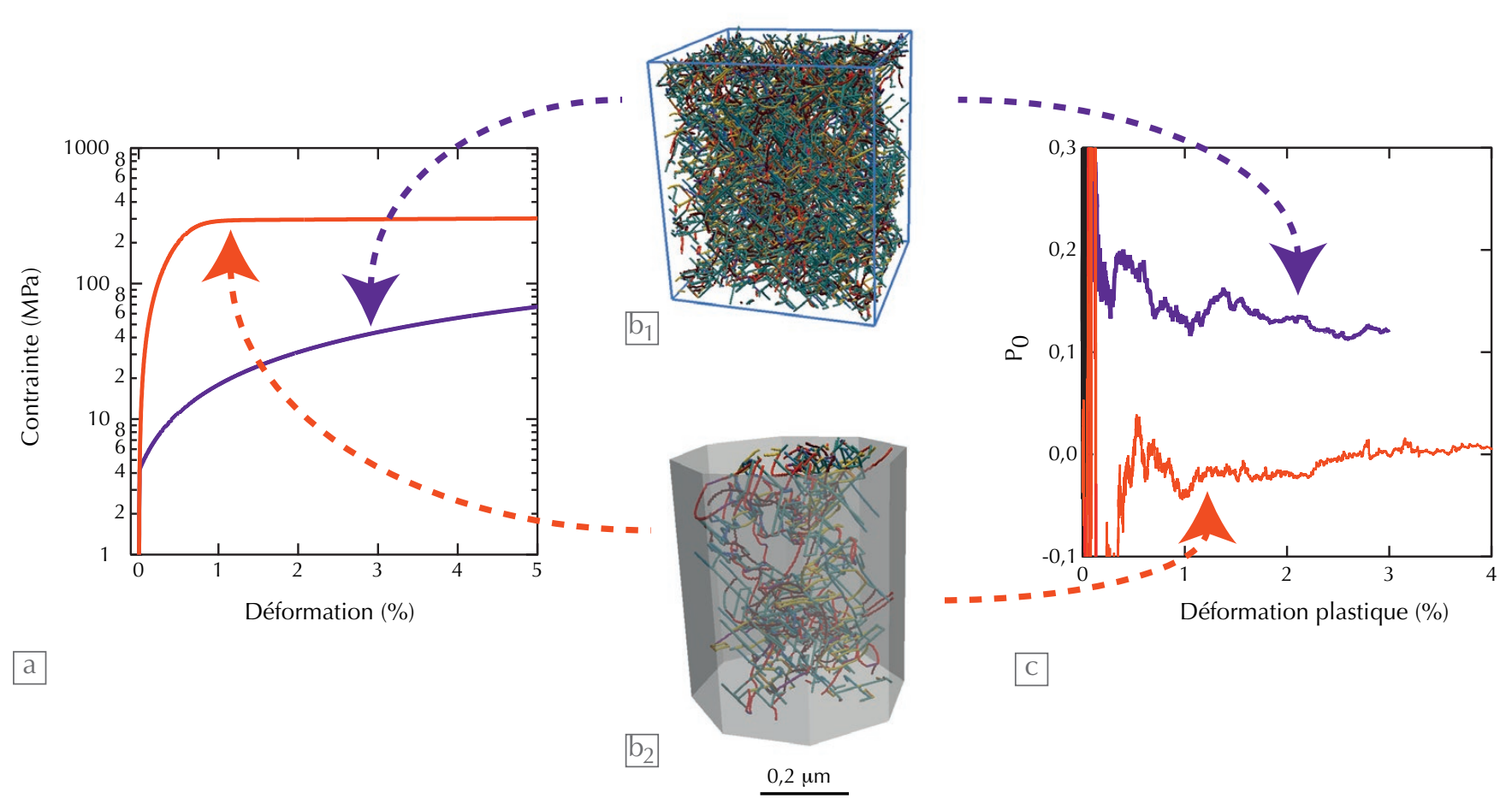

4. Effet de taille en plasticité cristalline.

Les propriétés plastiques des cristaux changent avec leur taille (a). Un petit élément de volume, partie d'un cristal de grande dimension (bł), se déforme sous des contraintes beaucoup plus faibles qu'un micro-objet fait du même matériau et de même dimension ( $b_{2}$ ). Dans les deux cas, la déformation plastique est intermittente ; cependant, le libre parcours moyen des dislocations augmente rapidement dans les micro-objets. Cette évolution résulte essentiellement d'une diminution significative du taux de formation de jonctions $\mathrm{P}_{0}$, qui décroît rapidement avec la déformation dans des objets submicroniques. Ce phénomène empêche l'accumulation de dislocations dans le cristal (c). C'est pourquoi les objets de toute petite dimension perdent leur capacité d'écrouissage.

\section{$>>$}

des simulations de DD est relativement immédiat et permet le développement de modèles macroscopiques mieux justifiés physiquement, utilisant un nombre réduit de paramètres pertinents.

Ainsi, il devient possible de modéliser quantitativement les propriétés mécaniques macroscopiques de matériaux simples, tels que des métaux purs, à partir de lois de comportement génériques. L'exemple reproduit en figure 4 est représentatif de cette démarche multi-échelle. Dans un échantillon de taille finie, la déformation plastique est influencée par la présence de surfaces libres qui attirent les dislocations à l'extérieur du cristal pour minimiser l'énergie élastique. Ces forces de surface sont à l'origine d'un fort effet de taille, découvert il y a une vingtaine d'années. Ainsi, lorsque l'on diminue la taille d'un échantillon jusqu'à des dimensions inférieures à la dizaine de micromètres, celui-ci d'une part devient de plus en plus dur et d'autre part se déforme plastiquement avec de moins en moins d'écrouissage. Comme illustré sur la figure 4 , cet effet de taille peut encore être décrit avec les lois de comportement conventionnelles de la plasticité des matériaux massifs. Les simulations de DD peuvent cependant nous renseigner sur l'évolution avec la taille de l'échantillon, des paramètres physiques contrôlant la contrainte critique de formation des avalanches de dislocations et le libre parcours moyen des dislocations.

\section{Remarques finales}

De nouveaux ponts entre l'étude des propriétés dynamiques des dislocations et la mécanique des matériaux s'établissent grâce à des concepts statistiques anciens, comme le libre parcours moyen des dislocations. Si A est l'aire totale balayée par une avalanche, sa dimension linéaire caractéristique est approximativement $\mathrm{A}^{1 / 2}$. La distribution des amplitudes d'avalanches pendant un essai de déformation restant bornée, il est alors possible de définir une taille moyenne d'avalanche $<\mathrm{A}^{1 / 2}>$, fonction des conditions de sollicitation mécanique. A priori, le libre parcours moyen des dislocations et la taille moyenne des avalanches sont donc deux quantités physiques reliées entre elles par un facteur de proportionnalité. La découverte de liens, tels que celui-ci, reliant les aspects discret et continu de la plasticité cristalline, est un enjeu essentiel. Il est probable que ces progrès vont permettre dans les années à venir le développement de nouvelles méthodes de caractérisation des matériaux, non destructives, plus rapides et plus sensibles aux détails microstructuraux.

\section{Références}

1• L. Kubin, Dislocations, mesoscale simulations and plastic flow, dans 0xford Series on Materials Modelling, volume 5, Oxford University Press (2013).

2• J. Friedel, Dislocations, Pergamon Press, 0xford (1967).

3• M. Zaiser, "Scale invariance in plastic flow of crystalline solids", Advances in Physics, 55 (2006) 185-245.

4 B. Devincre, T. Hoc et L. Kubin, “Dislocation mean free paths and strain hardening of crystals", Science, 320 (2008) 1745-1748. 